\title{
ON FIGURES OF EQUILIBRIUM OF A ROTATING COMPRESSIBLE FLUID MASS; CERTAIN NEGATIVE RESULTS*
}

BY

\section{E. J. MOULTON}

1. Introduction. The problem of determining from theoretical considerations the shape of celestial bodies has led to extensive discussions of figures of equilibrium of a rotating fluid mass. Especial difficulties have been encountered when the fluid is assumed compressible, the case in nature. They are in fact so great that various writers have considered it worth while to establish that certain figures, for a certain type of law of density of the fluid mass, are not figures of equilibrium. Thus Hamy t proved that an ellipsoidal mass composed of a finite number of layers of homogeneous fluid in which the density increases layer by layer from surface to center and the bounding surfaces of the layers are ellipsoids coaxial with the surface ellipsoid, cannot be a figure of equilibrium. Véronnet $\ddagger$ extended Hamy's theorem to the case in which the density is any continuous function increasing from surface to center. Volterra§ arrived at the same negative conclusion, supposing the equidensity surfaces to be homothetic ellipsoids, and the density function to be any positive, finite, integrable function not a constant.

In this paper other negative theorems are established. Surfaces of great generality are considered, as contrasted with the ellipsoidal surfaces discussed by earlier writers, but generality in the conclusion is lost in so far as there may be for a given surface a finite number of values of a certain compressibility parameter for which the conclusion is not true. The surfaces discussed include all ellipsoids, and the results for ellipsoids are new since it is not assumed that equidensity surfaces are ellipsoids.

2. Notation and fundamental equations. A fluid mass is considered whose surface is $\Sigma$ and density at any point $P$ is $\rho$; such a mass is referred to briefly

\footnotetext{
* Presented to the Society, under a slightly different title, December 27, 1913.

† Thesis (Paris), Étude de la figure des corps célestes, 1887. Reproduced by Tisserand, Traité de Mécanique Céleste, vol. 2, pp. 186-192.

‡ Thesis (Paris), Rotation de l'ellipsoide hétérogène et figure exacte de la Terre, 1912, J o u r $\mathrm{n}$ a 1 d e M a thém a tiques, ser. 6, vol. 8 (1912), pp. 331-465.

§ Sur la stratification d'une masse fluide en équilibre, A c t a $\mathrm{M}$ a t $\mathrm{h}$ e $\mathrm{m}$ a t i c a , 27 (1903), pp. 105-124.
} 
as the mass $(\Sigma, \rho)$. The mass is rotating with an angular velocity $\omega$ about an axis $A$; the angular velocity is constant throughout the mass. The only forces acting arise from the mutual attractions of the particles under the newtonian law, and from the internal pressure.

In order for the fluid mass to be in equilibrium the following conditions must be satisfied:

I. At every point of the fluid

$$
d p=f \rho d U,
$$

where $d p$ and $d U$ are the differentials of the pressure and of the potential of the attraction and centrifugal force, and where $f$ is the gravitation constant.

II. On the surface $\Sigma$ the potential $U$ and the density $\rho$ are constant.

III. The center of gravity of the mass $(\Sigma, \rho)$ lies on the axis $A$ of rotation.

If, for a mass $(\Sigma, \rho)$ rotating with an angular velocity $\omega$ about an axis $A$, conditions I, II, III are satisfied, then $(\Sigma, \rho, \omega, A)$ will be called a system of equilibrium.

Turning to the equations of the problem, we have first

$$
U=\frac{\omega^{2} R^{2}}{2 f}+\int \frac{\rho d \tau}{\Delta},
$$

where $R$ is the distance from the axis of rotation $A$ to the point $P$ at which $U$ is computed, and where $\Delta$ is the distance from $P$ to the volume element $d \tau$, and the integral is extended throughout the mass $(\Sigma, \rho)$. Next we assume a relation between the pressure and density in the mass to hold, expressed by the equation

$$
h d p=f \rho \frac{\partial F(\rho, h)}{\partial \rho} d \rho,
$$

where $h$ is a compressibility parameter, and $F(\rho, h)$ is a polynomial* in $\rho$, is analytic in $h$ in the vicinity of $h=0$, and is su'ch that

if $\rho_{0}>0, \rho_{0}$ being a constant.

$$
\left.\frac{\partial F(\rho, h)}{\partial \rho}\right]_{\rho=\rho_{0}} \neq 0,
$$

Equations (1) and (3) are satisfied if and only if

$$
\frac{\partial F(\rho, h)}{\partial \rho} d \rho=h d U \quad \text { or } \quad \rho=0 .
$$

The latter equation may be discarded in our problem. The solutions of the former under the condition that $\rho=\rho_{0}>0$ at a fixed point $P_{0}$ in the mass $(\Sigma, \rho)$ are considered; they are solutions of

$$
F\left(\rho_{1}, h\right)=F\left(\rho_{0}, h\right)+h\left(U_{1}-U_{0}\right),
$$

\footnotetext{
* The function $F(\rho, h)$ may be greatly generalized in the later argument; see $\S 7$.
} 
where subscripts are used to indicate the point at which the functional values are taken, $P_{1}$ being a variable point in the mass $(\Sigma, \rho)$. In view of equation (2), we may write (4) in the form

$$
\begin{gathered}
F\left(\rho_{1}, h\right)=F\left(\rho_{0}, h\right)+\frac{h \omega^{2}}{2 f}\left(R_{1}^{2}-R_{0}^{2}\right)+h \int \kappa_{12} \rho_{2} d \tau_{2}, \\
\kappa_{12}=\frac{1}{\Delta_{12}}-\frac{1}{\Delta_{02}},
\end{gathered}
$$

where $\Delta_{12}$ is the distance from $P_{1}$ to the volume element $d \tau_{2}$, and $\Delta_{02}$ has a similar meaning.

Equation (5) is the fundamental integral equation which the density function must satisfy. It has its simplest form when $F(\rho, h)=\rho$. In this case (3) is the Laplace pressure-density equation*

and (5) may be written

$$
h d p=f \rho d \rho ;
$$

$$
\rho_{1}^{\prime}=1+h q\left(R_{1}^{2}-R_{0}^{2}\right)+h \int \kappa_{12} \rho_{2}^{\prime} d \tau_{2},
$$

$$
\rho_{1}^{\prime}=\rho_{1} / \rho_{0}, \quad q=\omega^{2} / 2 f \rho_{0} .
$$

3. More exact statement of the problem. The general problem of determining figures of equilibrium from our point of view may now be stated thus: Given an axis of rotation $A$ and a pressure-density equation of the form (3), it is required to find an angular velocity $\omega$, a surface $\Sigma$, and a constant $\rho_{0}>0$, such that a solution $\rho$ of equation (5) makes $(\Sigma, \rho, \omega, A)$ a system of equilibrium. Our method will be to make the compressibility parameter $h$ play a fundamental rôle; that is, various functions will be expanded as power series in this parameter. In this paper $\dagger$ we shall let $\Sigma$ be any one of an extensive class of surfaces and $P_{0}$ any interior point, and consider the possibility of determining $\omega$ and $\rho_{0}$ as functions of $h$ such that when equation (5) is satisfied $(\Sigma, \rho, \omega, A)$ is a system of equilibrium for some continuous range of values of $h$. The negative conclusion we shall reach shows that to solve the problem by our method it is necessary to let $\Sigma$ vary with $h$.

In order to describe $\Sigma$ accurately, we make the following definitions. By a regular surface we understand one which can be cut into a finite number of pieces, each of which has at every point a continuously turning tangent plane; by a simple surface, one which is continuous and has no multiple points or lines. The surface $\Sigma$ will be chosen from the class of closed, simple, regular surfaces.

We shall assume that $\omega$ and $\rho_{0}$ can be determined as desired and derive

* The pressure-density equation $d p=h \rho d \rho$ was used by Laplace (Mécanique Céleste, book XI, chap. IV); it is to be observed that our $h$ is proportional to the reciprocal of the Laplace $h$. For us, $h=0$ gives an incompressible fluid.

$\dagger$ In a later paper I propose to adopt a different method, and let $\Sigma$ vary with $h$. 
necessary consequences. We first consider the special case when (5) reduces to the simpler equation $\left(5^{\prime}\right)$.

4. The solution of the integral equation $\left(5^{\prime}\right)$. This is a linear integral equation of the Fredholm type, but presents an unusual difficulty in the fact that the kernel $\kappa_{12}$ becomes infinite not only when the variable points $P_{1}$ and $P_{2}$ coincide, but also when $P_{2}$ coincides with the fixed point $P_{0}$. This difficulty is met by considering the iterated equation*

$$
\rho_{1}=\eta_{1}+h^{2} \int \kappa_{12}^{(2)} \rho_{2} d \tau_{2},
$$

where we have dropped accents and written

$$
\begin{aligned}
\eta_{1} & =1+h q\left(R_{1}^{2}-R_{0}^{2}\right)+h \int \kappa_{12}\left[1+h q\left(R_{2}^{2}-R_{0}^{2}\right)\right] d \tau_{2}, \\
\kappa_{12}^{(2)} & =\int \kappa_{13} \kappa_{32} d \tau_{3} \\
& =\int\left[\frac{1}{\Delta_{13} \Delta_{32}}-\frac{1}{\Delta_{03} \Delta_{32}}\right] d \tau_{3}-\frac{1}{\Delta_{02}} \int\left[\frac{1}{\Delta_{13}}-\frac{1}{\Delta_{03}}\right] d \tau_{3} .
\end{aligned}
$$

The function $\eta_{1}$ is readily seen to be continuous in $P_{1}$ throughout $\Sigma$; moreover the last two integrals are continuous functions $\dagger$ in $P_{1}$ and $P_{2}$ throughout $\Sigma$, and hence we may write $\ddagger$

$$
\kappa_{12}^{(2)}=\mu_{12}+\mu_{12}^{\prime} \cdot \frac{1}{\Delta_{02}}
$$

where the only discontinuity comes in through the factor $1 / \Delta_{02}$. If we use polar coördinates with the pole at $P_{0}$, we have

$$
\Delta_{02}=r_{2}, \quad d \tau_{2}=r_{2}^{2} \sin \theta_{2} d r_{2} d \theta_{2} d \phi_{2},
$$

and hence the function $\eta_{1}$ and the kernel $\kappa_{12}^{(2)} r_{2}^{2} \sin \theta_{2}$ are continuous in $\left(r_{1}, \theta_{1}, \phi_{1}\right)$ and $\left(r_{1}, \theta_{1}, \phi_{1}, r_{2}, \theta_{2}, \phi_{2}\right)$ respectively. Thus the apparent difficulty in equation (7), due to the way in which the kernel becomes infinite, disappears.

We know then from the general theory of integral equations, $\S$ that equa-

* That is, the equation obtained from $\left(5^{\prime}\right)$ by changing the subscripts 1 and 2 to 2 and 3 and substituting the right member for $\rho_{2}$ in the integral of $\left(5^{\prime}\right)$, and then altering the notation slightly. It is here, where an interchange of order of integration occurs, and later in this article in connection with solving (7), that some assumption concerning the character of $\Sigma$ is chiefly needed. It could be of less restricted class.

$\dagger$ The continuity of the function given by the first integral follows from the inequalities given by Fredholm, A c t a $\mathrm{M}$ a the m a t i c a, vol. 27 (1903), p. 387. The second integral is the potential for a constant density, which is known to be continuous.

$\ddagger$ As a matter of fact $\mu_{12}^{\prime}$ is independent of $P_{2}$.

\$ See, for example, Bôcher, An Introduction to the Study of Integral Equations; or Lalesco, Introduction d la Théorie des Équations Intégrales. 
tion (7) has, except for isolated finite values of $h$, a unique solution continuous in $P_{1}$, and it readily follows that the same is true of $\left(5^{\prime}\right)$. Also we may write the solution

$$
\rho_{1}=\eta_{1}+h^{2} \int K_{12}^{(2)} \eta_{2} d \tau_{2},
$$

where $K_{12}^{(2)}$ is, except for isolated finite values of $h$, analytic in $h$ and continuous in $P_{1}$ and $P_{2}$ throughout $\Sigma$. In particular, $K_{12}^{(2)}$ is analytic in $h$ at $h=0$.

By use of equation (8) we write

$$
\rho_{1}=1+h q\left(R_{1}^{2}-R_{0}^{2}\right)+h S_{1}(1)+h^{2} q S_{1}\left(R^{2}-R_{0}^{2}\right),
$$

where, if $f$ is any function whose value at $P_{i}$ is $f_{i}$,

$$
S_{1}(f)=\int \kappa_{12} f_{2} d \tau_{2}-h \int K_{12}^{(2)}\left[f_{2}+h \int \kappa_{23} f_{3} d \tau_{3}\right] d \tau_{2} .
$$

We observe that when $f$ is independent of $h, S_{1}(f)$ is analytic in $h$ except for isolated finite values of $h$, and in particular is analytic at $h=0$.

5. The first conclusion. If $\omega$ and $\rho_{0}$ can be determined so that when $\rho_{1}$ is a solution of $\left(5^{\prime}\right)(\Sigma, \rho, \omega, A)$ is a system of equilibrium for other than isolated finite values of $h$, then it is possible to determine $q\left(=\omega^{2} / 2 f \rho_{0}\right)$ so that the function $\rho_{1}$ given by (10) is effective in satisfying equilibrium conditions II and III. In the first place $q$ may be so chosen that the values of $\rho$ at any two points $P_{1}$ and $P_{2}$ on $\Sigma$ are the same. The value of $q$ for this purpose is readily found to be

$$
q=\frac{S_{2}(1)-S_{1}(1)}{R_{1}^{2}-R_{2}^{2}+h\left[S _ { 1 } \left(R_{1}^{2}-\frac{\left.\left.R_{0}^{2}\right)+S_{2}\left(R_{1}^{2}-R_{0}^{2}\right)\right]}{},\right.\right.}
$$

where subscripts on the $S$ 's indicate the points at which they are computed. Since $q\left(=\omega^{2} / 2 f \rho_{0}\right)$ is constant as far as $P_{1}$ and $P_{2}$ are concerned, its characterization as to $h$ is obtained by choosing $P_{1}$ and $P_{2}$ so that $R_{1} \neq R_{2}$. We thus find that $q$ as given by (11) is analytic in $h$ except for isolated finite values, and in particular is analytic at $h=0$. This value of $q$, therefore, when substituted in (10), determines a function $\rho$ which is in general analytic in $h$, in particular in the vicinity of $h=0$, and which for all such values of $h$ is constant on $\Sigma$. Also since the center of gravity of $(\Sigma, \rho)$ is on the axis $A$ for other than isolated finite values of $h$, it is in general on $A$, and in particular for all values of $h$ near $h=0$.

The argument thus far reduces the consideration of values of $h$ to those in the vicinity of $h=0$. We now write

$$
\rho_{1}=\sum_{i=0}^{\infty} \rho_{1}^{(i)} h^{i} ; \quad q=\sum_{i=0}^{\infty} q^{(i)} h^{i},
$$


where $\rho_{1}^{(i)}$ is continuous throughout $\Sigma$. Equilibrium conditions I and II require (a) that $\rho_{\mathrm{I}}^{(i)}$ be constant on $\Sigma$, and (b) that the center of gravity of $\left(\Sigma, \rho^{(i)}\right)$ lie on the axis $A$.

By substituting (12) into $\left(5^{\prime}\right)$ and equating coefficients of like power of $h$, we find that

$$
\begin{aligned}
& \rho^{(0)}=1, \\
& \rho^{(i)}=q^{(i-1)}\left(R_{1}^{2}-R_{0}^{2}\right)+\int \kappa_{12} \rho_{2}^{(i-1)} d \tau_{2} \quad(i=1,2,3, \cdots) .
\end{aligned}
$$

The right member of (13) is, except for an added constant, the potential at $P_{1}$, for a certain angular velocity, of the mass $\left(\Sigma, \rho^{(i-1)}\right)$. In view of the italicized statement in the preceding paragraph, taking $i=1$, we now have the following negative result.

Theorem 1. Let an axis $A$ of rotation and a closed, simple, regular surface* $\Sigma$ be chosen arbitrarily. Then, (a) if $\Sigma$ is not an equipotential surface of the mass $(\Sigma, 1)$ when rotating with a suitable constant angular velocity, or $(b)$ if the center of gravity of the homogeneous mass $(\Sigma, 1)$ is not on $A$, there exist only isolated finite values of $h$ for which $\omega$ and $\rho_{0}$ may be so chosen that, if $\rho$ is a continuous solution of $\left(5^{\prime}\right),(\Sigma, \rho, \omega, A)$ is a system of equilibrium.

6. The case of an ellipsoid. If $\Sigma$ is an ellipsoid and $A$ is a diameter, the center of gravity of the mass $(\Sigma, 1)$ lies on $A$, but $\Sigma$ may or may not be an equipotential surface of $(\Sigma, 1)$ when rotating with a proper angular velocity; if it is not, Theorem 1 applies; if it is, we proceed one step further.

For this purpose, we use the following method of computing the newtonian potential. Let $\Sigma$ be

$$
\frac{x^{2}}{a^{2}}+\frac{y^{2}}{b^{2}}+\frac{z^{2}}{c^{2}}=1
$$

and let us write

$$
\mu=1-\frac{x^{2}}{a^{2}}-\frac{y^{2}}{b^{2}}-\frac{z^{2}}{c^{2}} ; \quad \nu=1-\frac{x^{2}}{a^{2}+s}-\frac{y^{2}}{b^{2}+s}-\frac{z^{2}}{c^{2}+s} .
$$

Then if the density is constant on the surface $\mu=$ const., the newtonian potential is given by $\dagger$

$$
V=\int_{0}^{\infty} \int_{0}^{\nu} \rho(\mu) d \mu \frac{d s}{D(s)}, \quad D(s)=\frac{\sqrt{\left(a^{2}+s\right)\left(b^{2}+s\right)\left(c^{2}+s\right)}}{\pi a b c} .
$$

Now suppose $P_{0}$ is taken on the axis $A$, so that $R_{0}=0$. Taking $i=1$

* A more extensive class of surfaces might be used; e. g., $\Sigma$ might be composed of a finite number of non-intersecting closed, simple, regular surfaces, and the conclusion still be true.

† This formula was used by Volterra, loc. cit., p. 108, with a slightly different notation. The proof is readily made by comparison with pp. 138-140 of Mathieu's Théorie du Potential, where a similar formula is derived for the potential at an exterior point. 
in (13), we have then from (15)

$$
\rho_{1}^{(1)}=q^{(0)} R_{1}^{2}-M_{1} x_{1}^{2}-M_{2} y_{1}^{2}-M_{3} z_{1}^{2},
$$

where

$$
\begin{gathered}
M_{1}=\int_{0}^{\infty} \frac{d s}{\left(a^{2}+s\right) D(s)}, \quad M_{2}=\int_{0}^{\infty} \frac{d s}{\left(b^{2}+s\right) D(s)}, \\
M_{3}=\int_{0}^{\infty} \frac{d s}{\left(c^{2}+s\right) D(s)} .
\end{gathered}
$$

It is readily proved that since $\rho_{1}^{(1)}$ is constant on $\Sigma_{1}$, and hence the right member of (16) is constant in $x_{1}$ and $y_{1}$ when $z_{1}$ is eliminated by use of (14), the axis $A$ of rotation must be a principal axis of $\Sigma$. Suppose $A$ is the $z$-axis. On eliminating $z_{1}$ from (16) and equating the coefficients of $x_{1}^{2}$ and $y_{1}^{2}$ to zero, we have

$$
q^{(0)}-M_{1}-\frac{c^{2}}{a^{2}} M_{3}=0, \quad q^{(0)}-M_{2}-\frac{c^{2}}{b^{2}} M_{3}=0 .
$$

The ellipsoid must therefore have axes so related that for some value of $q^{(0)}$ these two equations hold simultaneously. ${ }^{*}$ For such ellipsoids

$$
\rho_{1}^{(1)}=-c^{2} M_{3}\left[\frac{x_{1}^{2}}{a^{2}}+\frac{y_{1}^{2}}{b^{2}}+\frac{z_{1}^{2}}{c^{2}}\right]=c^{2} M_{3}(\mu-1)
$$

We consider next $\rho^{(2)}$. From (13) we have

$$
\rho_{1}^{(2)}=q^{(1)} R_{1}^{2}+\int \kappa_{12} \rho_{2}^{(1)} d \tau_{2} .
$$

The integral is, except for an added constant, the newtonian potential for the density function $\rho_{2}^{(1)}$. It may therefore be computed by use of (15) and (17). It is seen that $\rho_{1}^{(2)}$ contains terms of second and fourth degrees in $x_{1}, y_{1}, z_{1}$; the latter are

$$
\frac{c^{2} M_{3}}{2} \int_{0}^{\infty}\left(\frac{x_{1}^{2}}{a^{2}+s}+\frac{y_{1}^{2}}{b^{2}+s}+\frac{z_{1}^{2}}{c^{2}+s}\right)^{2} \frac{d s}{D(s)} .
$$

When $z_{1}$ is eliminated by use of (14) the fourth degree terms must vanish; hence

$$
\int_{0}^{\infty} \frac{\left(a^{2}-c^{2}\right) s d s}{\left(a^{2}+s\right)\left(c^{2}+s\right) D(s)}=0 ; \quad \int_{0}^{\infty} \frac{\left(b^{2}-c^{2}\right) s d s}{\left(b^{2}+s\right)\left(c^{2}+s\right) D(s)}=0 .
$$

Hence we have $a=b=c$; that is $\Sigma$ is a sphere. Hence

THEOREM 2. Even if $\Sigma$ is an ellipsoid, not a sphere, and $A$ is a diameter of $\Sigma$, the conclusion of Theorem 1 is true.

* These conditions are satisfied if the ellipsoid is of revolution or if it is one of the figures of equilibrium discovered by Jacobi-the Jacobian ellipsoids. 
7. The more general pressure-density equations. In the preceding paragraphs our equations come from the initial use of the Laplace pressure-density equation $\left(3^{\prime}\right)$. We now consider the consequences of the initial use of the more general equation (3). We choose the surface $\Sigma$ and axis $A$ as in the preceding discussion and attempt the formal determination of $\rho_{0}, \rho$, and $\omega^{2}$ as functions analytic in $h$ in the vicinity of $h=0$ such that equation $\left(5^{\prime}\right)$ and equilibrium conditions II and III are satisfied for all values of $h$ in the vicinity of $h=0$. If we write

$$
\rho_{0}=\sum_{i=0}^{\infty} \rho_{0}^{(i)} h^{i} ; \quad \rho=\sum_{i=0}^{\infty} \rho^{(i)} h^{i} ; \quad \frac{\omega^{2}}{2 f}=\sum_{i=0}^{\infty} \omega^{(i)} h^{i},
$$

conditions II and III require that $\rho^{(i)}$ be constant on $\Sigma$ and that the center of gravity of the mass $\left(\Sigma, \rho^{(i)}\right)$ be on $A$.

If we substitute (18) in (5) and take the value at $h=0$ of $F\left(\rho_{1}, h\right)$ and its first and second $h$-derivatives, ${ }^{*}$ we have

$$
\begin{gathered}
F\left(\rho_{1}^{(0)}, 0\right)=F\left(\rho_{0}^{(0)}, 0\right), \\
\frac{\partial F\left(\rho_{1}^{(0)}, 0\right)}{\partial \rho_{1}} \cdot \rho_{1}^{(1)}+\frac{\partial F\left(\rho_{1}^{(0)}, 0\right)}{\partial h}=\frac{\partial F\left(\rho_{0}^{(0)}, 0\right)}{\partial \rho_{0}} \cdot \rho_{0}^{(1)}+\frac{\partial F\left(\rho_{0}^{(0)}, 0\right)}{\partial h} \\
+\omega^{(0)}\left(R_{1}^{2}-R_{0}^{2}\right)+\int \kappa_{12} \rho_{2}^{(0)} d \tau_{2}, \\
\frac{\partial F\left(\rho_{1}^{(0)}, 0\right)}{\partial \rho_{1}} \cdot \rho_{1}^{(2)}+\frac{\partial^{2} F\left(\rho_{1}^{(0)}, 0\right)}{\partial \rho_{1}^{2}} \cdot \rho_{1}^{(1)^{2}}+2 \frac{\partial^{2} F\left(\rho_{1}^{(0)}, 0\right)}{\partial \rho_{1} \partial h} \rho_{1}^{(1)} \\
+\frac{\partial^{2} F\left(\rho_{1}^{(0)}, 0\right)}{\partial h^{2}}=\frac{\partial F\left(\rho_{0}^{(0)}, 0\right)}{\partial \rho_{0}} \rho_{0}^{(2)}+\frac{\partial^{2} F\left(\rho_{0}^{(0)}, 0\right)}{\partial \rho_{0}^{2}} \cdot \rho_{0}^{(1)^{2}} \\
+2 \frac{\partial^{2} F\left(\rho_{0}^{(0)}, 0\right)}{\partial \rho_{0} \partial h} \rho_{0}^{(1)}+\frac{\partial^{2} F\left(\rho_{0}^{(0)}, 0\right)}{\partial h^{2}}+2 \omega^{(1)}\left(R_{1}^{2}-R_{0}^{2}\right) \\
+\int \kappa_{12} \rho_{2}^{(1)} d \tau_{2} .
\end{gathered}
$$

Equation $(19 a)$ is satisfied by $\rho_{1}^{(0)}=\rho_{0}^{(0)}$, which makes $\rho_{1}$ equal $\rho_{0}$, as it should, when the point $P_{1}$ coincides with $P$. Since $\rho_{0}^{(0)}$ is a constant, $\rho_{1}^{(0)}$ is constant on $\Sigma$, satisfying one equilibrium condition. The center of gravity condition requires that the center of gravity of the mass $\left(\Sigma, \rho_{0}^{(0)}\right)$, and hence of $(\Sigma, 1)$, lie on the axis $A$. Solving equation $(19 b)$ for $\rho_{1}^{(1)}$, we have a constant added to a constant multiple of

$$
\omega^{(0)} R_{1}^{2}+\int \kappa_{12} \rho_{2}^{(0)} d \tau_{2} .
$$

* It is only in computing these derivatives that we need restrictions on the kind of function $F(\rho, h)$ may be. Any function which leads to equations $(19 a),(19 b),(19 c)$ on differentiating as to $h$, and for which the coefficient of $\rho_{1}^{(1)}$ and $\rho_{1}^{(2)}$ is not zero, may be used. 
This expression is therefore constant on $\Sigma$ under precisely the same conditions as the function $\rho_{1}^{(1)}$ of the preceding paragraphs, and differs from that function only by an added and a multiplied constant. Hence when $\Sigma$ is an ellipsoid and $A$ is a diameter of $\Sigma$, the function $\rho_{1}^{(1)}$ is either not constant on $\Sigma$ or it is given by a formula similar to (17):

$$
\rho_{1}^{(1)}=C_{1} \mu+C_{2}, \quad C_{1} \neq 0 .
$$

When we consider $\rho_{2}^{(2)}$, as found from (19c), we see that, since $\rho_{1}^{(1)}$ is constant on $\Sigma, \rho_{2}^{(2)}$ is constant on $\Sigma$ if and only if

$$
2 \omega^{(1)} R_{1}^{2}+\int \kappa_{12} \rho_{2}^{(1)} d \tau_{2}
$$

is constant on $\Sigma$. This leads at once, as before, to the conclusion $a=b=c$. Hence we have

Theorem 3. Let $\Sigma$ and $A$ be as described in Theorem 1. Under the same hypotheses (a), (b), or the hypothesis of Theorem 2, it is impossible to determine $\omega, \rho_{0}$, and $\rho$ as power series in $h$ so that equation (5) shall be satisfied, $\rho$ shall be constant when $h=0$, and $(\Sigma, \rho, \omega, A)$ shall be a system of equilibrium for all values of $h$ near $h=0$. 